\title{
Opinión del estudiantado de la Universidad de Zaragoza sobre las competencias docentes del profesorado universitario
}

\section{Opinion of the Students of the University of Zaragoza on Teaching skills of Higher Education Teachers}

\section{Concepción Bueno García, María-Isabel Ubieto-Artur, Ana Rosa Abadía Valle}

\section{Resumen}

En esta investigación se presentan los resultados de una encuesta realizada al profesorado y estudiantado de la Universidad de Zaragoza, relativa a las competencias que debe tener un buen docente universitario. Para ello se han distinguido seis competencias docentes: la interpersonal, metodológica, comunicativa, de planificación y gestión de la docencia, de trabajo en equipo y de innovación. Los resultados se centran prioritariamente en la opinión de los estudiantes, analizados según curso y rama de conocimiento de la titulación y se realiza una comparación entre las valoraciones otorgadas por los docentes y discentes a la importancia de las competencias presentadas. Entre las conclusiones se destaca que los estudiantes, independientemente del curso y de la rama de conocimiento de su titulación, coinciden en otorgar la mayor importancia para ser un buen docente a que este explique bien los contenidos de la asignatura y a que motive a los estudiantes. La competencia comunicativa suscita el acuerdo entre el profesorado y el alumnado en su valoración como la más importante.

Palabras Clave

Competencias del docente; Enseñanza Superior; Universidad de Zaragoza (España); Encuesta

\begin{abstract}
The research presents the results of a survey conducted among teachers and students of the University of Zaragoza, about the skills of a good university teacher. The skills studied have been six: interpersonal, methodological, communicative, teaching planning and management, teamwork and innovation. This paper focuses on the results of the students' opinion, according to their academic years and their knowledge branches. A comparison between the assessments of the teachers and those of the students about the importance of the analyzed competences is also established. Among the conclusions outstands that students, regardless their knowledge branches or their academic years, agree in granting the highest rating to the clear explanation of the subject's content and to the encouragement of motivation, in order to be a good teacher. So that teachers and students agree in assessing the communicative competence as the most important
\end{abstract}

Keywords

Skills of teachers; Higher education; University of Zaragoza (Spain); Survey 


\section{Introducción}

La declaración conjunta de los ministros europeos de enseñanza, de junio de 1999, marcó un hito en la educación superior. Previamente, en la declaración de La Sorbona de 1998 ya se había hecho "hincapié en la construcción del espacio europeo de educación superior como instrumento clave en la promoción de la movilidad de los ciudadanos, su empleabilidad, y el desarrollo global del continente". "Las instituciones europeas de enseñanza superior - aceptaron - el reto y - asumieron un papel principal en la construcción del espacio europeo de enseñanza superior". Sus principales objetivos estuvieron relacionados, entre otros, con la comparabilidad de los títulos, el establecimiento de un sistema de créditos (ECTS) para promover la movilidad de los estudiantes y “la promoción de una colaboración europea en la garantía de calidad con vistas al diseño de criterios y metodologías comparables" (Declaración de Bolonia, 1999).

Posteriormente se sucedieron diversos encuentros y comunicados, entre los que se encuentran Praga (2001), Berlín (2003), Bergen (2005), Londres (2007), Lovaina (2009), Budapest y Viena (2010) y Bucarest (2012), que fueron perfilando ese Espacio Europeo de Educación Superior (EEES), que ha venido a suponer un cambio en el paradigma educativo de la educación superior. La última reunión de los Ministros de Educación del EEES, celebrada en Ereván (Armenia), el 14 y 15 de mayo de 2015, en la que aprobaron los "Estándares y directrices para el aseguramiento de la calidad en el Espacio Europeo de Educación Superior (ESG)", ratifica ese cambio de paradigma cuando señala que "Las universidades deben garantizar que los programas se implementen de tal manera que promuevan que los alumnos adopten un papel activo en la creación de los procesos de aprendizaje y que la correspondiente evaluación refleja este enfoque" (Comunicado de Ereván, 2015).

De acuerdo con Fernández March (2006, pp. 39-40):

Los rasgos característicos de este nuevo modelo educativo exigen el desarrollo de un perfil profesional, de unos roles y unas actividades diferentes a las tradicionales en los estudiantes y los profesores. El perfil apropiado del estudiante viene caracterizado por los siguientes elementos: aprendiz activo, autónomo, estratégico, reflexivo, cooperativo, responsable. Sin duda, esto exige un gran cambio de mentalidad en la cultura dominante del alumnado universitario y una atención especial. En el caso de los profesores, tal y como se afirma en el informe de la investigación realizada por el equipo de Valcárcel (2003), un requisito básico para el logro de algunos de los objetivos del proceso de convergencia es la profesionalización del profesor universitario. Esta afirmación se traduce en la exigencia de una formación pedagógica institucionalizada y sistemática, cuya finalidad sea la de facilitar el aprendizaje de sus nuevas competencias docentes. 


\title{
2. Competencias docentes del profesorado
}

Para abordar el tema de las competencias docentes del profesorado debemos comenzar por definir brevemente qué entendemos por ello. Existen diferentes publicaciones de autores que han estudiado en profundidad este tema, como Zarifian (1999), Perrenoud (2004) o Cano (2010), pero destacaremos el concepto de competencia, que recoge el Proyecto Tuning y que afirma que:

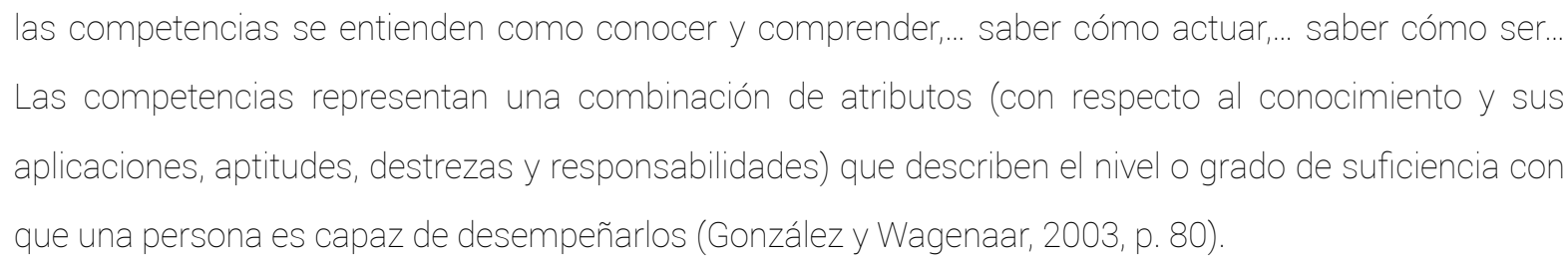

En cuanto a las competencias docentes del profesorado universitario, también han sido objeto de estudio por parte de diversos autores (Zabalza, 2003; Valcárcel, 2005; Tejada, 2009; Mas y Tejada, 2013). En 2012, la Revista de Docencia Universitaria (REDU) dedicó un número monográfico a las Competencias docentes en la Educación Superior, pues, como indican en su presentación, "las nuevas directrices educativas derivadas de la convergencia de los estudios universitarios en el Espacio Europeo, demandan del profesorado la adquisición de nuevos conocimientos y la actualización de las capacidades requeridas para la transmisión del saber" (Torra y Esteban, 2012, p. 17).

Siguiendo a Zabalza (2001, p. 71):

\begin{abstract}
tomamos el concepto de competencia como un constructo molar que nos sirve para referirnos al conjunto de conocimientos y habilidades que los sujetos necesitamos para desarrollar algún tipo de actividad. Obviamente, cada actividad suele exigir la presencia de un número variado de competencias que pueden posteriormente ser desglosadas en unidades más específicas de competencia en las que se especifican las tareas concretas que están incluidas en la competencia global... ...Por ejemplo, en la competencia "evaluar" una de las unidades de competencia puede ser el "preparar los exámenes" o "corregir los exámenes", etc.
\end{abstract}

Con este marco de referencia conceptual, Zabalza (2001) señala las siguientes competencias docentes del profesorado:

1. Planificar el proceso de enseñanza-aprendizaje.

2. Seleccionar y preparar los contenidos disciplinares.

3. Ofrecer informaciones y explicaciones comprensibles y bien organizadas (competencia comunicativa). 
4. Manejo de las nuevas tecnologías.

5. Diseñar la metodología y organizar las actividades.

6. Comunicarse-relacionarse con los alumnos.

7. Tutorizar.

8. Evaluar.

9. Reflexionar e investigar sobre la enseñanza.

10. Identificarse con la institución y trabajar en equipo.

Por otra parte, los nuevos retos de la formación del profesorado universitario llevaron a la creación del Grupo Interuniversitario de Formación Docente (GIFD), constituido por las unidades y centros de formación de las universidades públicas catalanas "con el fin de definir un perfil competencial y profesional del docente universitario y establecer un marco de referencia común que le permitiese elaborar planes de formación inicial y continua que ayuden al profesorado universitario a abordar su tarea docente con mayores garantías de éxito" (Torra, et al., 2012, p. 23). Las competencias docentes identificadas por este grupo fueron las siguientes:

1. Competencia interpersonal (CI): Promover el espíritu crítico, la motivación y la confianza, reconociendo la diversidad cultural y las necesidades individuales, creando un clima de empatía y compromiso ético.

2. Competencia metodológica (CM): Aplicar estrategias metodológicas (de aprendizaje y evaluación) adecuadas a las necesidades del estudiantado, de manera que sean coherentes con los objetivos y los procesos de evaluación, y que tengan en cuenta el uso de las tecnologías de la información y la comunicación (TIC) para contribuir a mejorar los procesos de enseñanza-aprendizaje.

3. Competencia comunicativa (CC): Desarrollar procesos bidireccionales de comunicación de manera eficaz y correcta, lo cual implica la recepción, interpretación, producción y transmisión de mensajes a través de canales y medios diferentes y de forma contextualizada a la situación de enseñanza-aprendizaje.

4. Competencia de planificación y gestión de la docencia (CPGD): Diseñar, orientar y desarrollar contenidos, actividades de formación y de evaluación, y otros recursos vinculados a la enseñanzaaprendizaje, de forma que se valoren los resultados y se elaboren propuestas de mejora.

5. Competencia de trabajo en equipo (CTE): Colaborar y participar como miembro de un grupo, asumiendo la responsabilidad y el compromiso propios hacia las tareas y funciones que se 
tienen asignadas para la consecución de unos objetivos comunes, siguiendo los procedimientos acordados y atendiendo los recursos disponibles.

6. Competencia de innovación (CDI): Crear y aplicar nuevos conocimientos, perspectivas, metodologías y recursos en las diferentes dimensiones de la actividad docente, orientados a la mejora de la calidad del proceso de enseñanza-aprendizaje. (Torra, et al., 2012, p. 30).

Posteriormente, en el marco del Proyecto de la Red de Docencia Universitaria REDU se llevó a cabo un Proyecto de Investigación, titulado "Propuesta de un marco de referencia competencial del profesorado universitario y adecuación de los planes de formación basados en competencias docentes" (Proyecto REDU 2012), donde se abordó un estudio más amplio, que, entre otros aspectos, incluía el análisis de las competencias docentes en el contexto social y profesional actual. En dicho proyecto participaron 15 universidades españolas, entre las que se encontraba la Universidad de Zaragoza (UZ). En él se recabó información relativa a la opinión que tenía tanto del profesorado como el estudiantado acerca de las competencias docentes de los primeros (Pagès, 2014).

El objetivo del presente trabajo es presentar y analizar la opinión que los docentes y discentes de primer y segundo ciclo de la Universidad de Zaragoza tienen sobre las competencias que debería tener un buen profesor universitario.

\section{Metodología}

Desde el punto de vista metodológico, se ha realizado un tratamiento diferenciado del profesorado y del alumnado. Al profesorado se le ha administrado una encuesta validada por el grupo GIFD. En el marco del Proyecto REDU 2012, se diseñó el cuestionario dirigido a los estudiantes con el objetivo de conocer la perspectiva de los mismos sobre las competencias docentes, previamente definidas por el GIFD. Para dirigirse al estudiantado, no se utilizaron, como en el estudio realizado entre los profesores, los indicadores de cada una de las competencias, sino que se reformularon las preguntas realizadas a los estudiantes, estableciéndose una relación entre estas y las competencias establecidas. Con el objeto de facilitar la comprensión de los mismos y aumentar el número de respuestas posibles, se reformuló el cuestionario, acortándolo y adaptándolo a un lenguaje menos científico.

Ambas encuestas se completaron en el curso 2010-2011 y tienen una estructura similar, incluyendo el perfil-sociodemográfico del encuestado, distintas cuestiones relacionadas con la importancia de las competencias o de los indicadores que las conforman, así como con la priorización de competencias y la posibilidad de incluir sus aportaciones en un formato de pregunta abierta. Las dos encuestas 
se encuentran disponibles en la Memoria final del Proyecto (Pagès, 2014, pp. 149-163 y 164-168 respectivamente).

La relación entre las competencias que valoró el profesorado y las preguntas hechas a los estudiantes se exponen en la Tabla 1.

\begin{tabular}{|c|c|}
\hline Competencias (C) & Preguntas $(\mathrm{P})$ \\
\hline \multirow{3}{*}{ Competencia Interpersonal (Cl) } & Estimular la reflexión crítica (P1) \\
\hline & Fomentar un clima de confianza y tolerancia en el aula (P2) \\
\hline & Motivar al alumnado en su proceso de aprendizaje (P3) \\
\hline \multirow{4}{*}{ Competencia Metodológica (CM) } & $\begin{array}{l}\text { Utilizar métodos de enseñanza-aprendizaje coherentes con } \\
\text { los objetivos de la asignatura (P4) }\end{array}$ \\
\hline & $\begin{array}{l}\text { Utilizar procedimientos de evaluación coherentes con los } \\
\text { objetivos de la asignatura (P5) }\end{array}$ \\
\hline & Fomentar la participación y el trabajo colaborativo (P6) \\
\hline & $\begin{array}{c}\text { Proporcionar feedback sobre el proceso de aprendizaje y } \\
\text { favorecer su reorientación (P7) }\end{array}$ \\
\hline \multirow{2}{*}{ Competencia Comunicativa (CC) } & Explicar de forma clara los contenidos de la asignatura (P8) \\
\hline & Escuchar a los estudiantes (promover el diálogo) (P9) \\
\hline \multirow{4}{*}{$\begin{array}{c}\text { Competencia de Planificación y Gestión de la } \\
\text { Docencia (CPGD) }\end{array}$} & Informar de la planificación de la asignatura (P10) \\
\hline & $\begin{array}{c}\text { Destacar los contenidos más relevantes en la titulación y } \\
\text { en la profesión (P11) }\end{array}$ \\
\hline & $\begin{array}{l}\text { Especificar claramente los criterios y los sistemas de } \\
\text { evaluación (P12) }\end{array}$ \\
\hline & Cumplir con el programa/guía de la asignatura (P13) \\
\hline \multirow{2}{*}{ Competencia de Trabajo en Equipo (CTE) } & Coordinarse con otros profesores de la asignatura (P14) \\
\hline & Coordinarse con el profesorado de otras asignaturas (P15) \\
\hline Competencia de Innovación (CDI) & $\begin{array}{c}\text { Introducir innovaciones que ayuden a mejorar el proceso de } \\
\text { enseñanza/aprendizaje (P16) }\end{array}$ \\
\hline
\end{tabular}

Tabla 1. Relación entre competencias docentes y preguntas realizadas a los estudiantes.

La encuesta se diseñó mediante herramientas distintas, según estuviera dirigida al profesorado o al estudiantado. Para los primeros se utilizó la plataforma Encuestafácil (bit.ly/21An22Q), que permite obtener de manera regular copias de seguridad e informes de participación detallados para el análisis posterior. Para los segundos, se usó la herramienta JotForm (http://www.jotform.com), que permite crear formularios en línea optimizados para dispositivos móviles, opción que podría resultar más atractiva para este colectivo. El diseño permitía a los estudiantes ofrecer su opinión, de forma anónima, sobre las características que definen a un buen docente.

Ambas encuestas se difundieron a través de distintos medios. Para los profesores se utilizaron el boletín informativo diario de la UZ (iUnizar) y los mensajes de correo electrónico personales, distribuidos por el Instituto de Ciencias de la Educación de la UZ. A los estudiantes se les pidió su colaboración a 
través del boletín informativo diario de la UZ (iUnizar) y por medio de sus representantes en el Consejo de Gobierno de la UZ. Además se solicitó la colaboración del Presidente del Consejo de Estudiantes de la Universidad. Todos ellos fomentaron la cumplimentación de las encuestas a través de distintas redes sociales.

El análisis de los datos se ha realizado calculando las medidas de tendencia central y de variabilidad de cada una de las preguntas. Para el estudio de las diferencias entre las medias por curso y por rama de conocimiento se ha utilizado el análisis de la varianza (ANOVA) y el test HSD de Tukey para las comparaciones múltiples.

Según los datos aportados por el Ministerio de Educación Cultura y Deporte, el número total de PDI de la Universidad de Zaragoza en el curso 2009/2010 fue de 3563, por lo que el tamaño de la muestra recomendado para un margen de error del 5\%, 95\% de nivel de confianza y $50 \%$ de nivel de heterogeneidad era de 347. La misma fuente nos informa de que el número de estudiantes de grado, primer y segundo ciclo de la Universidad de Zaragoza en el curso 2010/2011, fue de 30731 y el número de estudiantes de máster de 2021. El tamaño de muestra recomendada para cada uno de los grupos de estudiantes con los mismos parámetros de margen de error y niveles de confianza y heterogeneidad son 379 para los primeros y 323 para los estudiantes de Máster. El número de profesores que respondió a la encuesta fue de 414, y el de estudiantes de grado o licenciatura de la UZ fue de 2626. Corresponden, respectivamente, al 11,6\% y al 8,54\% del total de las poblaciones respectivas y ambas muestras son representativas.

La misma encuesta fue respondida por los estudiantes de máster en la Universidad de Zaragoza, de los que se recogieron 234 respuestas. Este número supone el $72,44 \%$ de la muestra estimada inicialmente para que los datos fuesen representativos. Por este motivo, la muestra no es estadísticamente representativa, y lo expuesto en cuanto a las valoraciones de los estudiantes de máster, constituye un análisis de tendencias para la reflexión.

\section{Resultados y discusión}

Los resultados de las encuestas realizadas al profesorado han sido presentados en las VII Jornadas de Innovación Docente e Investigación Educativa de la UZ y tienen su correspondiente publicación (Bueno, Ubieto-Artur y Abadía, 2015a). Por otra parte, algunos de los datos correspondientes a la valoración que hacen los estudiantes han sido comunicados en el Congreso Internacional sobre Aprendizaje, Innovación y Competitividad, CINAIC, 2015 (Bueno, Ubieto-Artur y Abadía, 2015b).

En el caso de los profesores, el $51 \%$ de los que respondieron la encuesta fueron hombres y el 49\%, mujeres. Debe tenerse en cuenta que en el curso 2010-2011 la población de PDI de la UZ se distribuía 
de la siguiente manera, atendiendo al sexo: el 59,43\% eran hombres y el 40,57\% mujeres. Sin embargo, en el caso de los estudiantes de grado y licenciatura, el 63\% de los que cumplimentaron la encuesta eran mujeres y el $37 \%$ hombres. Hay que destacar que el citado curso, el 55,43\% del estudiantado de grado de la UZ eran mujeres y el $44,57 \%$ hombres.

La media de edad de esta muestra de profesores es de 55 años y su desviación típica de 14,29, lo que nos da cuenta de una muestra envejecida y heterogénea en cuanto a la edad. Con respecto a los estudiantes de grado y licenciatura la media de edad se sitúa en 22,88 años y su desviación típica es de 6,13. Por tanto, si tenemos en cuenta el recorrido de la variable, podemos afirmar que, en cuanto a la edad, la muestra es bastante homogénea.

Si se tiene en cuenta la categoría profesional del profesorado, el porcentaje de respuestas más elevado corresponde a profesores titulares (39,5\%), seguido de profesores asociados (27,2\%). Según los datos ofrecidos por el Instituto Aragonés de Estadística, los profesores titulares en el curso 2009/2010 en la UZ eran el 32,6\% del total, mientras que los asociados suponían el 22,7\%. Igualmente, en el colectivo de PDI son las dos categorías profesionales más numerosas. El resto de las respuestas fueron realizadas por profesores ayudantes doctores $(11,7 \%)$, catedráticos $(7,6 \%)$ y profesores contratados doctores $(6,4 \%)$.

En cuanto a la experiencia como docente universitario, la mayoría del profesorado que respondió las encuestas (41,8\%) manifestaba contar con más de 15 años de experiencia y solo el 14,6\% señalaron tener menos de 3 años de experiencia docente.

La distribución por áreas de conocimiento del profesorado es la siguiente: en primer lugar, se encuentra el profesorado de Ciencias Sociales y Jurídicas (34\%), seguido por el de Ingeniería y Arquitectura y Ciencias de la Salud (19\% en ambos casos), el de Ciencias (17\%), siendo el colectivo menos numeroso el profesorado de Arte y Humanidades, que corresponde al 11\% de la muestra.

La distribución de los estudiantes por rama de conocimiento sigue un patrón similar: los más numerosos son los de Ciencias Sociales y Jurídicas (33,1\%), seguidos de los de Ingeniería y Arquitectura (22\%). Los de Ciencias de la Salud son un 20,9\%, los de Ciencias un 12,8\% y los de Arte y Humanidades un 13\%. Esta distribución es similar a la de la población de estudiantes de grado o licenciatura en el curso 2010/11 en la UZ.

Los estudiantes que han respondido al cuestionario y se sitúan siguiendo un único curso son 2455. La distribución de estos a lo largo de los cursos, de primero a cuarto, es relativamente uniforme: en primer y segundo curso hay algo más de un 19\%, en tercero sobre el $23 \%$ y en cuarto alrededor del 25\%. Los estudiantes de los dos cursos superiores correspondientes bien a licenciaturas en extinción, bien a grados de más de 4 cursos, son mucho menos numerosos, pues en quinto curso se sitúa algo 
más del 10\% y en sexto un 1,6\%.

Se analizan en primer lugar las calificaciones otorgadas a la importancia de las 16 características de un buen profesor, señaladas en la encuesta. Todas se sitúan entre el notable alto $(7,59)$ y el sobresaliente $(9,48)$. Por tanto, podemos afirmar que los estudiantes de la Universidad de Zaragoza conceden una gran importancia a todos los aspectos valorados. Además, la diferencia entre las puntuaciones medias mayor y menor es de 1,89 puntos sobre 10 (Tabla 2).

\begin{tabular}{|c|c|c|c|}
\hline Pregunta (P) & Media & Desviación Típica & Mediana \\
\hline P8 & 9,48 & 1,08 & 10 \\
\hline P3 & 9,31 & 1,13 & 9 \\
\hline P4 & 8,98 & 1,32 & 9 \\
\hline P5 & 8,94 & 1,35 & 9 \\
\hline P12 & 8,79 & 1,52 & 9 \\
\hline P2 & 8,7 & 1,5 & 9 \\
\hline P9 & 8,69 & 1,54 & 9 \\
\hline P10 & 8,66 & 1,58 & 9 \\
\hline P14 & 8,65 & 1,74 & 9 \\
\hline P1 & 8,53 & 1,57 & 9 \\
\hline P11 & 8,5 & 1,67 & 8 \\
\hline P16 & 8,17 & 1,7 & 8 \\
\hline P7 & 8,01 & 1,7 & 8 \\
\hline P13 & 7,9 & 1,93 & 8 \\
\hline P15 & 7,75 & 2,04 & 88 \\
\hline P6 & 7,59 & & \\
\hline
\end{tabular}

Tabla 2. Resumen de la valoración media de las preguntas de la encuesta de estudiantes de grado o licenciatura, ordenada de mayor a menor. Fuente: Elaboración propia (Bueno, et al., 2015b).

Si tenemos en cuenta la media y la desviación típica obtenidas, observamos que, en términos generales, aumenta la desviación típica al disminuir la media. Así, "explicar bien los contenidos" (P8) es lo más importante para los estudiantes de la Universidad de Zaragoza, pues otorgan una media de 9,48 puntos sobre 10 a este aspecto; la mitad de ellos ha puntuado con 10 y la desviación típica es la menor de las 16 cuestiones valoradas. Por el contrario, "fomentar la participación y el trabajo colaborativo" (P6) obtuvo la puntuación más baja $(7,59)$, con un menor grado de acuerdo, puesto que la desviación típica es de 1,98 y la mediana 8. 
Los estudiantes de la Universidad de Zaragoza, puntúan por encima de 9 dos características para ser un buen docente: "explicar de forma clara los contenidos de la asignatura" $(9,48)$ y "motivar al alumnado en su proceso de aprendizaje" $(9,31)$.

En el año 2014 se realizó una comparación de los datos obtenidos en la UZ con los datos globales de las universidades participantes en el Proyecto REDU 2012 (Bueno, et al., 2014), observándose puntuaciones similares en todos los casos. Al ordenar las puntuaciones medias otorgadas a cada uno de los 16 ítems de mayor a menor, encontramos coincidencias en el lugar que ocupan 12 de ellos. "Estímulo de la reflexión" (P1) ocupa el décimo lugar en los datos de UZ y el noveno en los datos globales. Por su parte, "fomentar la participación y el trabajo colaborativo" (P6) es el decimosexto en los datos de la UZ y el decimoquinto en los datos globales, "Coordinarse con otros profesores de la asignatura" (P14) es el noveno ítem en la UZ y el décimo para la muestra total. "Coordinarse con el profesorado de otras asignaturas" (P15) ocupa el último lugar en los datos de la UZ y el penúltimo en los datos globales.

\section{Opinión de los estudiantes de grado y licenciatura por ramas de conocimiento}

Al analizar los resultados según las ramas de conocimiento de las titulaciones de los estudiantes consultados (Tabla 3), observamos que igualmente otorgan una alta calificación a cada uno de los 16 ítems. De hecho, todos ellos obtienen una puntuación entre notable $(7,37)$ y sobresaliente $(9,6)$. Comparando las puntuaciones otorgadas por los estudiantes de cada rama de conocimiento de forma global, destacamos que los estudiantes matriculados en titulaciones de Ciencias de la Salud califican los distintos aspectos con puntuaciones más altas que las del resto. Por otra parte, los de titulaciones de Ciencias y de Ingeniería y Arquitectura son los que califican más bajo.

Aquí también se repite el orden de importancia concedida a las características del buen docente: los estudiantes de todas las ramas de conocimiento califican como más importante que los docentes "expliquen de forma clara los contenidos de la asignatura" (P8) y "motiven al alumnado en su proceso de aprendizaje" (P3).

La tercera característica que destacan los estudiantes de cada una de las ramas de conocimiento no coincide en todas ellas: Ciencias, Ciencias Sociales y Jurídicas e Ingeniería y Arquitectura consideran más importante que el docente "utilice métodos de enseñanza-aprendizaje coherentes con los objetivos de la asignatura" (P4), mientras que los de Arte y Humanidades valoran más que los docentes "estimulen la reflexión, la crítica y la autocrítica" (P1). Para los de Ciencias de la Salud "coordinarse con otros profesores de la asignatura" (P14) es el aspecto que valoran en tercer lugar. Sin embargo, esta coordinación está mucho menos valorada por los estudiantes de otras ramas de conocimiento, como es el caso de los de Arte y Humanidades, donde ocupa la décima posición. 


\begin{tabular}{|c|c|c|c|c|c|}
\hline & A H & C & C S & C S J & I A \\
\hline P1 & 9,06 & 8,4 & 8,54 & 8,6 & 8,24 \\
\hline P2 & 8,96 & 8,6 & 8,72 & 8,9 & 8,32 \\
\hline P3 & 9,31 & 9,25 & 9,4 & 9,37 & 9,18 \\
\hline P4 & 9,02 & 8,93 & 9,05 & 9 & 8,92 \\
\hline P5 & 8,96 & 8,9 & 9,09 & 8,95 & 8,78 \\
\hline P6 & 7,5 & 7,37 & 7,49 & 7,81 & 7,52 \\
\hline P7 & 8,03 & 7,79 & 8,08 & 8,22 & 7,77 \\
\hline P8 & 9,5 & 9,49 & 9,6 & 9,52 & 9,3 \\
\hline P9 & 8,69 & 8,72 & 8,73 & 8,86 & 8,41 \\
\hline P10 & 8,95 & 8,41 & 8,71 & 8,83 & 8,36 \\
\hline P11 & 8,25 & 8,23 & 8,88 & 8,58 & 8,33 \\
\hline P12 & 8,92 & 8,55 & 8,86 & 8,97 & 8,5 \\
\hline P13 & 7,87 & 7,72 & 8,39 & 7,84 & 7,64 \\
\hline P14 & 8,46 & 8,93 & 9,13 & 8,41 & 8,5 \\
\hline P15 & 7,55 & 8,03 & 8,39 & 7,44 & 7,56 \\
\hline P16 & 8,13 & 8,03 & 8,33 & 8,33 & 7,88 \\
\hline
\end{tabular}

Tabla 3. Puntuación media por ramas de conocimiento. En verde las puntuaciones más altas, en rojo las más bajas. AH: Arte y Humanidades, C: Ciencias, CS: Ciencias de la Salud, CSJ: Ciencias Sociales y Jurídicas, IA: Ingeniería y Arquitectura. Fuente: Elaboración propia (Bueno, et al., 2015b).

El aspecto menos valorado por los estudiantes es el "fomento del trabajo colaborativo" (P6) en todas las ramas de conocimiento excepto en Ciencias Sociales y Jurídicas, que lo ubican en penúltimo lugar. Para estos estudiantes, el ítem menos valorado es el de la "coordinación con el profesorado de otras asignaturas" (P15).

Estudiamos a continuación las diferencias entre las medias para cada una de las dieciséis preguntas utilizando el test de comparaciones múltiples HSD de Tukey. Se señala una diferencia estadísticamente significativa cuando el nivel de significación es de 0,05 (Tabla 4).

No se han encontrado diferencias estadísticamente significativas entre las medias otorgadas a la "importancia del uso de métodos de enseñanza-aprendizaje coherentes con los objetivos de la asignatura" (P4) para las distintas ramas de conocimiento. En todas las ramas se puntúa en torno a 9, siendo la única característica que concita esta unanimidad. Con respecto a la "utilización de procedimientos de evaluación coherentes con los objetivos de la asignatura" (P5) solamente se observan diferencias estadísticamente significativas entre los estudiantes de las titulaciones de Ciencias de la Salud e Ingeniería y Arquitectura. 
La cuestión que suscita mayores diferencias estadísticamente significativas entre las valoraciones de los estudiantes de las distintas ramas de conocimiento es "destacar los contenidos más relevantes en la titulación y en la profesión" (P11), junto con la de "coordinarse con otros profesores de la asignatura" (P14) (Tabla 4). Así, "destacar los contenidos más relevantes en la titulación y en la profesión" (P11) es la característica que valoran con una mayor puntuación los estudiantes de Ciencias de la Salud, seguidos por los de Ciencias Sociales y Jurídicas, cuya puntuación media es de 8,88 y 8,58 (Tabla 3). El caso de las medias otorgadas por los estudiantes de Ciencias de la Salud, posiblemente se deba al carácter marcadamente profesionalizante de estos estudios. "Coordinarse con otros profesores de la asignatura" (P14) resulta muy importante para los estudiantes de las ramas de Ciencias de la Salud $(9,13)$ y Ciencias $(8,93)$. No opinan de la misma manera los estudiantes de Ciencias Sociales y Jurídicas $(8,41)$ y de Arte y Humanidades $(8,46)$ (Tabla 3).

Por otra parte, "coordinarse con el profesorado de otras asignaturas" (P15) también presenta diferencias estadísticamente significativas entre dos grupos de ramas de conocimiento: el formado por Ciencias y Ciencias de la Salud y el de Arte y Humanidades, Ciencias Sociales y Jurídicas e Ingeniería y Arquitectura (Tabla 4).

Esta cuestión obtiene una calificación media alta y similar por parte del estudiantado de las ramas de Ciencias $(8,03)$ y Ciencias de la Salud $(8,39)$, mientras que los de las ramas restantes la consideran bastante memos importante, otorgándole una media en torno al 7,5 (Tabla 3).

La importancia media otorgada por los estudiantes de las titulaciones de Arte y Humanidades es mayor $(9,06)$ que la de las otras ramas con respecto a "estimular la reflexión, la crítica y la autocrítica" (P1), pues difiere del resto significativamente (Tabla 4). Destacan los estudiantes de titulaciones de Ingeniería y Arquitectura con la media más baja $(8,24)$ que es diferente a todas las demás, excepto a la media otorgada por los estudiantes matriculados en Ciencias (Tabla 3).

Encontramos una situación similar con respecto al "fomento de un clima de confianza y tolerancia en el aula" (P2), donde el estudiantado de Ingeniería y Arquitectura puntúa este ítem por debajo del resto $(8,32)$ y con diferencias estadísticamente significativas con respecto al resto de ramas de conocimiento (Tabla 4).

\begin{tabular}{|c|c|c|c|c|c|}
\hline & A H & C & C S & C S J & I A \\
\hline P1 & $a, b, d, g$ & $a$ & $b, i$ & $d, j$ & $g, i, j$ \\
\hline P2 & $a, g$ & $a, e, h$ & $i$ & $e, j$ & $g, h, i, j$ \\
\hline P3 & & & $i$ & $j$ & $i, j$ \\
\hline P4 & & & & & \\
\hline
\end{tabular}




\begin{tabular}{|c|c|c|c|c|c|}
\hline P5 & & & $i$ & & $i$ \\
\hline P6 & & $e$ & $f$ & $e, f, j$ & $j$ \\
\hline P7 & & $e$ & $i$ & $e, j$ & $i, j$ \\
\hline P8 & & $h$ & $i$ & $j$ & $i, j$ \\
\hline P9 & $a, g$ & $a, c, e$ & $c, i$ & $e, j$ & $h, i, j$ \\
\hline P10 & $b, d$ & $c, e$ & $b, c, f, i$ & $d, e, f, j$ & $i, j$ \\
\hline P11 & $a, g$ & $a, c, e$ & $c, i$ & $e, j$ & $g, i, j$ \\
\hline P12 & $b$ & $c$ & $b, c, f, i$ & $f$ & $i$ \\
\hline P13 & $a, b$ & $a, c, e, h$ & $b, c, f, i$ & $e, f$ & $h, i$ \\
\hline P14 & $a, b$ & $a, e, h$ & $b, f, i$ & $e, f$ & $h, i$ \\
\hline$P 15$ & & & $i$ & $j$ & $i, j$ \\
\hline$P 16$ & & & $i$ & $j$ \\
\hline
\end{tabular}

Tabla 4. Diferencias significativas en la valoración media seqún área de conocimiento. Diferencias significativas al 5\% para el test de HSD de Tukey: a: AH-C, b: AH-CS, c: C-CS, d: AH-CSJ, e: C-CSJ, f: CS-CSJ, g: AH-IA, h: C-IA, i: CS-IA y j: CSJ-IA.

Fuente: Elaboración propia (Bueno, et al., 2015b).

\section{Opinión de los estudiantes de grado y licenciatura por cursos}

Al observar las puntuaciones medias otorgadas por los estudiantes de grado y licenciatura por curso constatamos que la mayor es de 9,51 y la menor de 7,44 (Tabla 5). Un análisis general de los resultados nos permite afirmar que los estudiantes de primero puntúan más bajo que sus compañeros de cursos superiores. Posiblemente la experiencia adquirida a lo largo de los cursos les haga reconocer y valorar la importancia que estas características tienen para definir a un buen docente.

Una vez más existe unanimidad entre los estudiantes de todos los cursos, que consideran que las características más importantes para ser un buen docente son que "explique de forma clara los contenidos de la asignatura" (P8) y "motive al alumnado en su proceso de aprendizaje" (P3). Los estudiantes de primero, segundo y cuarto sitúan en tercer lugar, por orden de importancia, que el docente "utilice métodos de enseñanza-aprendizaje coherentes con los objetivos de la asignatura" (P4). Para los estudiantes de tercero la "utilización de procedimientos de evaluación coherentes con los objetivos de la asignatura" (P5) ocupa el tercer lugar y retrasan la característica anterior (P4) al duodécimo lugar (Tabla 5).

Considerando las respuestas por cursos, las preguntas P6 "Fomentar la participación y el trabajo colaborativo", P7 "Proporcionar feedback sobre el proceso de aprendizaje y favorecer su reorientación", P13 "Cumplir el programa/guía de la asignatura" y P15 "Coordinarse con el profesorado de otras 
asignaturas" son las menos valoradas por los estudiantes de los cuatro cursos. Estas son también las menos valoradas cuando atendemos a las respuestas de los estudiantes, según la rama de conocimiento de su titulación.

\begin{tabular}{|c|c|c|c|c|}
\hline & $\mathbf{1 0}^{\mathbf{0}}$ & $\mathbf{2}$ & $\mathbf{3}^{\mathbf{0}}$ & $\mathbf{4}^{\mathbf{0}}$ \\
\hline P1 & $\mathbf{8 , 3 8}$ & $\mathbf{8 , 4 7}$ & $\mathbf{8 , 4 2}$ & $\mathbf{8 , 6 4}$ \\
\hline P2 & $\mathbf{8 , 7 1}$ & 8,71 & 8,76 & $\mathbf{9}$ \\
\hline P3 & 9,28 & 9,34 & 9,33 \\
\hline P4 & 8,9 & 8,99 & 8,08 & 8,99 \\
\hline P5 & 8,76 & 8,97 & 9,03 & 8,97 \\
\hline P6 & 7,58 & 7,69 & 7,56 & 7,6 \\
\hline P7 & 7,72 & 8,11 & 8,05 & 8,21 \\
\hline P8 & 9,48 & 9,51 & 9,5 & 9,46 \\
\hline P9 & 8,69 & 8,85 & 8,74 & 8,67 \\
\hline P10 & 8,48 & 8,74 & 8,78 & 8,75 \\
\hline P11 & 8,3 & 8,56 & 8,57 & 8,58 \\
\hline P12 & 8,67 & 8,85 & 8,86 & 8,83 \\
\hline P13 & 7,89 & 8,08 & 7,93 & 8,87 \\
\hline P14 & 8,31 & 8,63 & 8,77 & 8,86 \\
\hline P15 & 7,44 & 7,67 & 7,94 & 8,01 \\
\hline P16 & 8,25 & 8,3 & 8,18 & \\
\hline
\end{tabular}

Tabla 5. Puntuación media otorgada por los estudiantes según curso. En verde las puntuaciones más altas, en rojo las más bajas. Fuente: Elaboración propia (Bueno, et al., 2015b).

Al utilizar el test HSD de Tukey para las comparaciones múltiples entre las puntuaciones medias otorgadas a cada pregunta por los estudiantes de los distintos cursos encontramos diferencias estadísticamente significativas entre los estudiantes de primero con respecto a los de tercero y los de cuarto en las preguntas: P1 "Estimular la reflexión crítica", P5 "Utilizar procedimientos de evaluación coherentes con los objetivos de la asignatura" y P11 "Destacar los contenidos más relevantes en la titulación y en la profesión" (Tabla 6).

Las medias delas respuestas a las preguntas P7"Proporcionar feedbacksobre el proceso deaprendizaje y favorecer su reorientación", P10 "Informar de la planificación de la asignatura", y P14 "Coordinarse con otros profesores de la asignatura" presentan diferencias estadísticamente significativas entre los estudiantes de primero y los de los demás cursos (Tabla 6). 
Por último, en P15 "Coordinarse con el profesorado de otras asignaturas" se han encontrado diferencias estadísticamente significativas entre los estudiantes de primero con los de tercero y cuarto y los de segundo con los de cuarto (Tabla 6).

La característica que valoran en menor medida los estudiantes de primer curso, significativamente más bajo que el resto de estudiantes, es la de "proporcionar feedback sobre el proceso de aprendizaje y favorecer su reorientación" (P7). Lo mismo ocurre con respecto a la "información sobre la planificación de la asignatura" (P10) y a la "coordinación con otros profesores de la asignatura" (P14).

\begin{tabular}{|c|c|}
\hline & Diferencias estadísticamente significativas \\
\hline P1 & $b, c$ \\
\hline P5 & $b, c$ \\
\hline P7 & $a, b, c, d$ \\
\hline P10 & $a, b, c, d$ \\
\hline P11 & $b, c$ \\
\hline P14 & a, b, c, d \\
\hline P15 & b, c, e \\
\hline
\end{tabular}

Tabla 6. Diferencias significativas en la valoración media según curso. Diferencias significativas al 5\% para el test de HSD de Tukey: a: $7^{0}-2^{\circ}$, b: $7^{0}-3^{\circ}, c: 7^{0}-4^{\circ}, d^{2}: 7^{0}-5^{\circ}$, e: $2^{0}-4^{\circ}$

\section{Opinión de los estudiantes de máster}

La misma encuesta fue respondida por los estudiantes de máster en la Universidad de Zaragoza, de los que se recogieron 234 respuestas. Como ya se ha señalado anteriormente, esta muestra no es estadísticamente representativa y por este motivo este apartado constituye un análisis de tendencias para la reflexión.

El 58,5\% de los estudiantes de máster que respondieron a la encuesta eran mujeres y el $41,5 \%$, hombres. En cuanto a rama de conocimiento del máster cursado, el 21,8\% pertenecían a Arte y Humanidades, el $13,2 \%$ a Ciencias, el $14,1 \%$ a Ciencias de la Salud, el 30,3\% a Ciencias Sociales y el $20,5 \%$ a Ingeniería y Arquitectura.

Al calcular las puntuaciones medias otorgadas a la importancia de las 16 características de un buen docente, destacamos que todas ellas son calificadas por encima del notable alto, alcanzando el sobresaliente, pues varían entre 7,67 y 9,34. Además, las medianas se sitúan entre 8 y 10, dato que nos corrobora la gran importancia otorgada a todas las características presentadas (Tabla 7).

Por su parte, las desviaciones típicas varían entre 1,83 y 1,16, concentrándose los valores más 
altos en las puntuaciones medias más bajas; es decir, en aquellos aspectos calificados como menos importantes, las valoraciones son más heterogéneas. Estos datos son coincidentes con los correspondientes a los estudiantes de grado y licenciatura.

En estudiantes de máster la pregunta P8 "Explicar de forma clara los contenidos de la asignatura" es la característica más valorada. Hay 5 aspectos valorados desde 9 hasta 9,34: el citado anteriormente además de P3 "Motivar al alumnado en su proceso de aprendizaje"; P1 "Estimular la reflexión, la crítica y la autocrítica"; P4 "Utilizar métodos de enseñanza-aprendizaje coherentes con los objetivos de la asignatura" y P5 "Utilizar procedimientos de evaluación coherentes con los objetivos de la asignatura".

\begin{tabular}{|c|c|c|c|}
\hline Pregunta (P) & Media & Desviación Típica & Mediana \\
\hline P8 & 9,34 & 1,16 & 10 \\
\hline P3 & 9,27 & 1,22 & 10 \\
\hline P1 & 9,18 & 1,25 & 9 \\
\hline P4 & 9,07 & 1,19 & 9 \\
\hline P5 & 9,00 & 1,37 & 9 \\
\hline P12 & 8,92 & 1,38 & 9 \\
\hline P2 & 8,82 & 1,48 & 9 \\
\hline P14 & 8,82 & 1,48 & 9 \\
\hline P10 & 8,77 & 1,45 & 9 \\
\hline P9 & 8,68 & 1,59 & 9 \\
\hline P7 & 8,59 & 1,58 & 9 \\
\hline P11 & 8,47 & 1,51 & 8 \\
\hline P16 & 8,16 & 1,79 & 8 \\
\hline P15 & 7,97 & 1,87 & 83 \\
\hline P6 & 7,94 & 1,90 & 8 \\
\hline P13 & 7,67 & & 9 \\
\hline
\end{tabular}

Tabla 7. Resumen de la valoración de los estudiantes de máster de las preguntas de la encuesta, ordenada de mayor a menor.

La mediana de las preguntas P8 "Explicar de forma clara los contenidos de la asignatura", P3 "Motivar al alumnado en su proceso de aprendizaje" y P1 "Estimular la reflexión, la crítica y la autocrítica" es 10, lo que ofrece una idea de la gran importancia que los estudiantes de máster otorgan a estos aspectos.

Los aspectos valorados por los estudiantes de máster por debajo de 8,5, son: "destacar los contenidos más relevantes en la titulación y en la profesión" (P11); "introducir innovaciones que ayuden a mejorar el proceso de enseñanza-aprendizaje" (P16); "coordinarse con el profesorado de otras asignaturas" 
(P15); "fomentar la participación y el trabajo colaborativo" (P6) y "cumplir el programa/guía de la asignatura" (P13).

\begin{tabular}{|c|c|c|}
\hline Pregunta & Prelación Máster & Prelación Grado \\
\hline P8 & 1 & 2 \\
\hline P3 & 2 & 10 \\
\hline P1 & 3 & 3 \\
\hline P4 & 4 & 4 \\
\hline P5 & 5 & 5 \\
\hline P12 & 6 & 6 \\
\hline P2 & 7 & 9 \\
\hline P14 & 8 & 8 \\
\hline P10 & 9 & 7 \\
\hline P9 & 10 & 13 \\
\hline P7 & 11 & 11 \\
\hline P11 & 12 & 12 \\
\hline P16 & 13 & 15 \\
\hline P15 & 14 & 16 \\
\hline P6 & 15 & 14 \\
\hline P13 & & \\
\hline
\end{tabular}

Tabla 8. Orden de prelación de las preguntas de la encuesta. En rojo aspectos a los que los estudiantes de máster conceden mayor prioridad que los de grado. En naranja aspectos a los que los estudiantes de grado conceden más importancia que los del máster

Comparando estos resultados con los obtenidos en el proyecto REDU 2012 (Abadía, et al., 2015), se observa que las tres características más valoradas (P8, P3, y P1) son las mismas. Entre las menos valoradas hay una pequeña diferencia, pues el orden que se obtiene en el estudio global es: P6, P15, P13, mientras que los estudiantes de máster de la UZ valoran en último lugar la pregunta P13, antecedida por P6 y la siguiente en orden ascendente es P15.

En tercer lugar, para los estudiantes de máster un buen docente debe "estimular la reflexión, la crítica y la autocrítica" (P1), mientras que los estudiantes de grado colocan esta característica en el décimo lugar. Esta es la diferencia más notable en el orden de importancia que otorgan los estudiantes de grado y máster. Es posible interpretar que estos últimos, al ser estudiantes de mayor edad, con una formación de grado o licenciatura, algunos incorporados ya al mundo laboral, valoran mucho más que el profesor, además de explicar con claridad y motivar, anime a que se pongan en juego capacidades más complejas como el pensamiento reflexivo o el crítico. 
Al comparar las características más valoradas por estudiantes de grado y máster, se observa coincidencia en las dos primeras: "explicar con claridad los contenidos de la asignatura" (P8) y "motivar al alumnado" (P3). Nos parece interesante recordar que estas características son las más importantes para los estudiantes de todas las ramas de conocimiento y de todos los cursos (Tabla 8).

La observación de la Tabla 8 nos permite afırmar que el resto de las diferencias en el orden de importancia otorgado por estudiantes de grado y máster son pequeñas: tan solo de una o dos posiciones.

Nos detenemos en los aspectos considerados menos importantes por los estudiantes de máster, correspondientes a las preguntas: P7 "Proporcionar feedback sobre el proceso de aprendizaje y favorecer su reorientación", P11 "Destacar los contenidos más relevantes en la titulación y en la profesión", P16 "Introducir innovaciones que ayuden a mejorar el proceso de enseñanza-aprendizaje", P15 "Coordinarse con el profesorado de otras asignaturas", P6 "Fomentar la participación y el trabajo colaborativo" y P13 "Cumplir el programa/guía de la asignatura". Encontramos que, aunque en distinto orden, también los estudiantes de grado colocan estas mismas características del buen profesor en los últimos lugares.

\section{Opinión de los estudiantes de máster por ramas de conocimiento}

En la Tabla 9 se reflejan las respuestas de los estudiantes de máster a estas 16 cuestiones sobre la importancia de tener ciertas habilidades y competencias para ser un buen docente, según la rama de conocimiento a la que están adscritos. La prueba Kruskal-Wallis de muestras independientes nos permite afırmar que hay diferencias estadísticamente significativas $(p<0,05)$ en las opiniones de los estudiantes según su rama de conocimiento en dos de ellas, concretamente en P1, "estimular la reflexión, la crítica y la autocrítica" y P13, "cumplir con el programa/guía de la asignatura".

A continuación se estudian las diferencias entre las medias para P1 "Estimular la reflexión, la crítica y la autocrítica" y P13 "Cumplir el programa/guía de la asignatura", mediante el test de comparaciones múltiples HSD de Tukey y se señala una diferencia estadísticamente significativa cuando el nivel de significación es de 0,05.

Se han encontrado diferencias estadísticamente significativas en cuanto a la importancia media otorgada por los estudiantes de máster a que el profesor "estimule la reflexión, la crítica y la autocrítica" entre los estudiantes de Ingeniería y Arquitectura $(8,5)$ con los de Ciencias Sociales y Jurídicas $(9,35)$ y con los de Arte y Humanidades $(9,61)$. En cuanto a esta característica, los estudiantes de máster de Ingeniería y Arquitectura no lo consideran tan importante como los estudiantes de Arte y Humanidades o los de Ciencias Sociales y Jurídicas. Este dato parece bastante coherente con la 
visión tradicional de las disciplinas, pero no está claro que esta visión sea la más realista.

El "cumplimiento del programa/guía de la asignatura" (P13) presenta también diferencias estadísticamente significativas en cuanto a las medias otorgadas por los estudiantes de Arte y Humanidades $(8,24)$ y los de Ciencias Sociales y Jurídicas $(7,31)$.

Para el resto de aspectos analizados no se han encontrado diferencias estadísticamente significativas, por lo que podemos afirmar que los estudiantes consideran cada uno de ellos de una importancia similar, independientemente de la rama a la que esté adscrito el máster que cursan.

En la Tabla 9 se recogen los valores medios para cada pregunta según ramas de conocimiento. Las características más importantes de un profesor para los estudiantes de las ramas de Ciencias, Ciencias de la Salud e Ingeniería y Arquitectura son que el profesor "explique con claridad los contenidos de la asignatura" (P8) y que "motive al alumnado" (P3). En las ramas de Arte y Humanidades y Ciencias Sociales y Jurídicas, aparece como mejor valoradas el "estímulo de la reflexión, la crítica y la autocrítica" (P1), seguida de "explicar con claridad los contenidos de la asignatura" (P8) y "motivar al estudiantado" (P3).

Las tres características consideradas menos importantes para los estudiantes de máster adscritos a las ramas de Ciencias de la Salud, Ciencias Sociales y Jurídicas e Ingeniería y Arquitectura son: "fomentar la participación y el trabajo colaborativo" (P6), "cumplir con el programa/guía de la asignatura" (P13) y "coordinarse con el profesorado de otras asignaturas" (P15).

Por su parte, los estudiantes de máster de la rama de Arte y Humanidades coinciden con los de las ramas anteriores en otorgar menos importancia a las preguntas en relación al "fomento de la participación y el trabajo colaborativo" (P6) y la "coordinación con profesorado de otras asignaturas" (P15). Sin embargo, para ellos "introducir innovaciones que ayuden a mejorar el proceso de enseñanzaaprendizaje" (P16) se encuentra entre las tres menos valoradas (Tabla 9).

En general, el grado de uso de las herramientas propias de XarFED por parte de los usuarios ha sido más bien bajo (con mayoría de valoraciones entre 0 y 2), aspecto que contrasta con la percepción de su grado de utilidad (mayoría de valoración entre 3 y 5), como se puede observar en las figuras 10 y 11, así como la Tabla 2. 


\begin{tabular}{|c|c|c|c|c|c|}
\hline & A H & C & C S & C S J & I A \\
\hline P1 & 9,61 & 9,16 & 9,12 & 9,35 & 8,5 \\
\hline P2 & 8,88 & 8,9 & 9,18 & 8,87 & 8,35 \\
\hline P3 & 9,33 & 9,42 & 9,3 & 9,34 & 9 \\
\hline P4 & 9,18 & 9,23 & 9,12 & 8,94 & 9,02 \\
\hline P5 & 9,22 & 8,84 & 8,91 & 8,9 & 9,08 \\
\hline P6 & 8,02 & 7,9 & 7,91 & 8,01 & 7,77 \\
\hline P7 & 8,41 & 8,26 & 8,85 & 8,9 & 8,38 \\
\hline P8 & 9,51 & 9,45 & 9,3 & 9,32 & 9,13 \\
\hline P9 & 8,96 & 9 & 8,55 & 8,7 & 8,25 \\
\hline P10 & 9,02 & 8,61 & 8,76 & 8,83 & 8,54 \\
\hline P11 & 8,55 & 8,42 & 9,06 & 8,35 & 8,21 \\
\hline P12 & 9,08 & 8,58 & 8,97 & 8,96 & 8,88 \\
\hline P13 & 8,24 & 7,71 & 8,12 & 7,31 & 7,27 \\
\hline P14 & 8,73 & 9,19 & 8,88 & 8,66 & 8,85 \\
\hline P15 & 7,71 & 8,52 & 8 & 8 & 7,85 \\
\hline P16 & 8,18 & 8,26 & 8,27 & 8,08 & 8,13 \\
\hline P16 & & & & 8 \\
\hline
\end{tabular}

Tabla 9. Puntuación media de estudiantes de máster por ramas de conocimiento. En verde puntuaciones más altas, en rojo las más bajas. AH: Arte y Humanidades, C: Ciencias, CS: Ciencias de la Salud, CSJ: Ciencias Sociales y Jurídicas, IA: Ingeniería Arquitectura.

A su vez, las tres habilidades consideradas menos importantes por los estudiantes de másteres de la rama de Ciencias, son la referida a la "puesta en marcha de innovaciones docentes" (P16), al "fomento de la participación y el trabajo colaborativo" (P6) y "cumplir con el programa guía de la asignatura" (P13) (Tabla 9).

\section{Aproximación a la comparación de la opinión de los estudiantes con la del profesorado}

La comparación de la opinión de los estudiantes con la del profesorado no resulta fácil, pues, como se ha señalado anteriormente, se han utilizado dos encuestas diferentes, que también eran valoradas de una forma diferente. En el caso del profesorado se preguntaba acerca de la importancia de cada una de las competencias (CI, CM, CC, CPGD, CTE y CDI) para el ejercicio de la profesión docente en la universidad, utilizando una escala de cuatro niveles correspondientes a 1. Nada, 2. Poca, 3. Bastante y 4. Mucha. Con respecto a cada una de las competencias se preguntaba por la importancia de cada uno de los elementos que las definen (Pagès, 2014). 
No obstante, los 16 ítems que valoraron los estudiantes están directamente relacionados con los elementos que configuran cada competencia docente, por lo que fue posible establecer una correspondencia entre las preguntas de la encuesta de los estudiantes (P1 a P16) y las competencias interpersonal $(\mathrm{Cl})$, metodológica (CM), comunicativa (CC), de planificación y gestión de la docencia (CPGD), trabajo en equipo (CTE) e innovación (CDI), correspondencia que se muestra en la Tabla 10. (Pagès, 2014).

\begin{tabular}{|c|c|c|c|}
\hline Competencia & Preguntas cuestionario estudiantes & $\begin{array}{c}\text { Importancia } \\
\text { profesorado }\end{array}$ & $\begin{array}{c}\text { Importancia } \\
\text { estudiantado }\end{array}$ \\
\hline $\mathrm{Cl}$ & $\mathrm{P} 1, \mathrm{P} 2, \mathrm{P3}$ & 4 & 2 \\
\hline $\mathrm{CM}$ & $\mathrm{P} 4, \mathrm{P} 5, \mathrm{P} 6, \mathrm{P} 7$ & 2 & 4 \\
\hline $\mathrm{CC}$ & $\mathrm{P} 8, \mathrm{P9}$ & 1 & 1 \\
\hline $\mathrm{CPGD}$ & $\mathrm{P} 10, \mathrm{P} 11, \mathrm{P} 12, \mathrm{P} 13$ & 3 & 5 \\
\hline $\mathrm{CTE}$ & $\mathrm{P} 14, \mathrm{P} 15$ & 6 & 6 \\
\hline $\mathrm{CDI}$ & $\mathrm{P} 16$ & 5 & 6 \\
\hline
\end{tabular}

Tabla 10. Relación entre las preguntas del cuestionario de los estudiantes y las competencias de la encuesta del profesorado y orden de prelación para cada colectivo.

El orden de importancia de las distintas competencias para el profesorado fue analizado por Bueno et al. (2015a), y para establecer el orden de las mismas para los estudiantes se ha tomado la media de los valores medios asignados a cada grupo de preguntas relacionado con cada competencia (valores reflejados en la Tabla 2 de este artículo).

Como se puede ver en la Tabla 10, tanto para el profesorado como para los estudiantes, la competencia comunicativa (CC) es la que tiene mayor relevancia; no en vano incluye la pregunta que consigue la mayor puntuación asignada por los estudiantes "explicar de forma clara el contenido de la asignatura" (P8).

Se observa discrepancia en cuanto a la segunda competencia más importante para los dos colectivos. Para el profesorado es más importante la competencia metodológica que la interpersonal (que sitúan en cuarto lugar), pero esta es la segunda más importante para los estudiantes, que precisamente relegan la competencia metodológica al cuarto puesto. Dentro de la competencia interpersonal (Cl) se ubica la pregunta P3 "Motivar al alumnado en su proceso de aprendizaje", que es la pregunta que los estudiantes colocan siempre en segundo lugar de importancia, sin distinguir si el análisis se hace agrupando las respuestas por el curso o por rama de conocimiento. 
Una de las posibles razones por las que la competencia metodológica (CM) se sitúa en cuarto lugar de importancia para el estudiantado de grado o licenciatura es que esta competencia incluye la pregunta que obtiene la menor puntuación de la encuesta de los estudiantes: "fomentar la participación y el trabajo colaborativo" (P6).

Tanto profesores como estudiantes coinciden en considerar en tercer lugar la competencia de planificación y gestión de la docencia (CPGD). Vuelve a observarse una diferencia en la valoración de la competencia trabajo en equipo (CTE), que es más importante para los estudiantes que la competencia innovadora (CDI), invirtiéndose de nuevo el orden en el caso del profesorado.

\section{Conclusiones}

En este trabajo se ha tomado el modelo competencial de Torra et al. (2012) que define seis competencias docentes del profesorado universitario: interpersonal, metodológica, comunicativa, de planificación y gestión de la docencia, de trabajo en equipo y de innovación.

El conjunto de competencias que se ha propuesto para su valoración en el estudio ha recibido altas puntuaciones tanto por el profesorado como por los estudiantes de grado, licenciatura y máster de la Universidad de Zaragoza.

El profesorado y el estudiantado de grado y licenciatura de la Universidad de Zaragoza coinciden en que la competencia comunicativa es la más importante para un buen docente universitario; la competencia en planificación y gestión de la docencia ocupa el tercer lugar y las competencias menos valoradas son la de trabajo en equipo y la de innovación docente. Las competencias interpersonal y metodológica plantean cierta división de opiniones entre profesorado y estudiantado, siendo la competencia metodológica más importante para el profesorado que la interpersonal, más valorada por el estudiantado.

Se han observado diferencias en la valoración de las distintas competencias en función de la rama de conocimiento de los estudiantes. Por ejemplo, los estudiantes de Ingeniería y Arquitectura ofrecen sistemáticamente unas puntuaciones más bajas que el resto de sus compañeros en todos los aspectos valorados.

Los estudiantes de primero valoran las características del buen docente con puntuaciones menores que las de sus compañeros de cursos superiores. Los estudiantes de tercero y cuarto curso valoran más que los de los cursos precedentes que el profesorado "proporcione feedback sobre el proceso de aprendizaje y favorezca su reorientación", "informe sobre la planificación de la asignatura" y se "coordine con otros profesores de la asignatura". 
La muestra de los estudiantes de máster nos permite describir tendencias. Este colectivo coincide con el de los estudiantes de grado y licenciatura en proponer como los aspectos más importantes del buen docente universitario que este "explique bien los contenidos de la asignatura" y que "motive al alumno". Otra característica de estos estudiantes es que consideran muy relevante el hecho de que el profesorado "estimule la reflexión, la crítica y la autocrítica".

Estas opiniones de los docentes y discentes universitarios sobre la importancia de las competencias del buen docente pueden aportar información valiosa a la hora de planificar la formación, así como de diseñar instrumentos de evaluación docente del profesorado universitario.

\section{Agradecimientos}

Este trabajo seha realizado en el marco del Proyecto “Propuesta de un marco de referencia competencial del profesorado universitario y adecuación de los planes de formación basados en competencias docentes", REDU 2012. Concepción Bueno García es miembro del Grupo de Investigación "EtnoEdu”. María Isabel Ubieto Artur y Ana Rosa Abadía Valle pertenecen al Grupo de Investigación en Docencia e Innovación Universitaria "GIDIU", ambos grupos, reconocidos por el Gobierno de Aragón.

Por su colaboración en el análisis de los datos a Teresa Pagès, Sarai Sabaté, M. ${ }^{a}$ Dolores Márquez y Helga Jorba.

\section{Referencias}

Abadía Valle, A. R., Bueno García, C., Ubieto-Artur, M. I., Márquez Cebrián, M. D., Sabaté, Díaz, S., Jorba Noguera, H. y Pagès Costa, T. (2015). Competencias del buen docente universitario. Opinión de los estudiantes. REDU - Revista de Docencia Universitaria, 13(2), 363-390. Recuperado de bit.ly/1p00mWU

Bueno, C., Ubieto-Artur, M. I. y Abadía, A. R. (2014). Características de un buen docente universitario: opinión de los estudiantes. En Universidad de Zaragoza. Vicerrectorado de Política Académica, Instituto de Ciencias de la Educación (coord.), Actas de las VIII Jornadas de Innovación Docente e Investigación Educativa. Zaragoza, 10 y 11 de Septiembre de 2014 (pp. 32-33). Recuperado de bit.ly/1QGVvRJ

Bueno, C., Ubieto-Artur, M. I. y Abadía, A. R. (2015a). ¿Qué competencias docentes debe tener el profesorado universitario? En Universidad de Zaragoza, Vicerrectorado de Política Académica, Instituto de Ciencias de la Educación (eds.), El trabajo en equipo, una herramienta para el aprendizaje (pp. 246250). Zaragoza: Universidad de Zaragoza, Vicerrectorado de Política Académica, Instituto de Ciencias de la Educación. Recuperado de bit.ly/21E30ks 
Bueno García, C., Ubieto-Artur, M. I. y Abadía Valle, A. R. (2015b). ¿Cómo es un buen docente para los estudiantes de la Universidad de Zaragoza? En A. Fidalgo-Blanco, M. L. Sein-Echaluce Lacleta, y F. J. García-Peñalvo (eds.), La Sociedad del Aprendizaje. Actas del III Congreso Internacional sobre Aprendizaje, Innovación y Competitividad. CINAIC 2015 (14-16 de Octubre de 2015, Madrid, España) (pp. 676-680). Madrid: Fundación General de la Universidad Politécnica de Madrid. Recuperado de bit. ly/1LxHgTw

Cano García, E. (2010). Cómo mejorar las competencias de los docentes. Guía para la autoevaluación y el desarrollo de las competencias del profesorado. Barcelona: Graó.

Comunicado de Ereván. (2015). Estándares y directrices para el aseguramiento de la calidad en el Espacio Europeo de Educación Superior (ESG) aprobados por la Conferencia de Ministros de Educación del EEES el 15 de mayo de 2015 (Ereván, Armenia). Recuperado de bit.ly/1TqTlaH

Declaración de Bolonia. (1999). The European Higher Education Area. Bologna Declaration. Joint Declaration of the European Ministers of Education. Bologna, 19 de junio de 1999. Recuperado de bit. ly/1QJ3KwP

Fernández March, A. (2006). Metodologías activas para la formación de competencias. Educatio Siglo $X X I, 24,35-56$.

González, J. y Wagenaar, R. (Eds.). (2003). Tuning Educational Structures in Europe. Informe Final. Fase Uno. Deusto: Universidad de Deusto. Recuperado de bit.ly/10JG5FO

Mas, O. y Tejada, J. (2013). Funciones y competencias en la docencia universitaria. Barcelona: Síntesis.

Pagès, T. (coord.). (2014). Propuesta de un marco de referencia competencial del profesorado universitario y adecuación de los plantes de formación basados en competencias docentes. Octubre 2014. Recuperado de http://goo.gl/SdHywV

Perrenoud, P. (2004). Diez nuevas competencias para enseñar. Barcelona: Graó.

Tejada, J. (2009). Competencias docentes. Profesorado. Revista de Currículum y Formación del Profesorado, 13(2). Recuperado de bit.ly/10Mx2UN

Torra, I., Corral, I. de, Pérez, M. J., Triadó, X., Pagès, T., Valdarrama, E., Màrquez, M. D., Sabaté, S., Solà, P., Hernàndez, C., Sangrà, A., Guàrdia, L., Estebanell, M., Patiño, J., González, A.P., Fandos, M., Ruiz, N., Iglesias, M. C., Tena, A. (2012). Identificación de competencias docentes que orienten el desarrollo de planes de formación dirigidos a profesorado universitario. REDU - Revista de Docencia Universitaria, 10(2), 21-56. Recuperado de bit.ly/24z5S4s 
Torra Bitlloch, I. y Esteban Moreno, R. M. (2012). Presentación. REDU - Revista de Docencia Universitaria, Número monográfico dedicado a Competencias docentes en la Educación Superior, 10(2), 17-20. Recuperado de bit.ly/1RDSXr1

Valcárcel, M. (Coord.). (2005). La preparación del profesorado universitario para la convergencia europea en educación superior (EA2003-0040). Informe Investigación. Programa de Estudios y Análisis del Ministerio de Ciencia e Innovación. Recuperado de bit.ly/1QJ89jp

Zabalza, M. A. (2001). La enseñanza universitaria: el escenario y sus protagonistas. Madrid: Narcea.

Zabalza, M. A. (2003). Competencias docentes del profesorado universitario. Calidad y desarrollo profesional. Madrid: Narcea.

Zarifian, P. (1999). Objectif compétence. Pour une nouvelle logique. Paris: Éditions Liaisons. 